\title{
Diseño Universal para el Aprendizaje (DUA) como estrategia pedagógica en
} educación inicial

\section{Universal Design for Learning (UDL) as a pedagogical strategy in initial education}

\author{
María Cecilia Palaguachi-Tenecela \\ maria.palaguachi @psg.ucacue.edu.ec \\ Universidad Católica de Cuenca, Azogues \\ Ecuador \\ https://orcid.org/0000-0003-1227-2102 \\ Darwin Gabriel Garcia-Herrera \\ dggarciah@ucacue.edu.ec \\ Universidad Católica de Cuenca, Azogues \\ Ecuador \\ https://orcid.org/0000-0001-6813-8100 \\ Sergio Constantino Ochoa-Encalada \\ scochoae@ucacue.edu.ec \\ Universidad Católica de Cuenca, Cuenca \\ Ecuador \\ https://orcid.org/0000-0003-3067-3719 \\ Juan Carlos Erazo-Álvarez \\ jcerazo@ucacue.edu.ec \\ Universidad Católica de Cuenca, Cuenca \\ Ecuador \\ https://orcid.org/0000-0001-6480-2270
}

Recibido: 24 de abril de 2020

Revisado: 02 de mayo de 2020

Aprobado: 20 de mayo de 2020

Publicado: 03 de junio de 2020

\section{RESUMEN}

La investigación tuvo como objetivo aplicar estrategias pedagógicas para niños y niñas de educación inicial basadas en el Diseño Universal para el Aprendizaje (DUA) con la finalidad de fortalecer la intervención del docente en los procesos de enseñanzaaprendizaje del C.E.I "Alonso Torres", mediante la planificación de actividades motivadoras. Se basó desde el enfoque cualitativo con método etnográfico con la 
finalidad de conocer y describir los hechos tal como se generaron en la realidad, de acuerdo a las perspectivas de los participantes. El cambio que requiere la educación está en las manos de los docentes, pues ellos deben ir a la par con las nuevas sociedades, todo depende de la actitud y el deseo de mejorar en beneficio de todos los niños y niñas, mediante actualizaciones y capacitaciones que permitan el conocimiento de nuevas estrategias como la forma de aplicarlas mejorando la calidad educativa.

Descriptores: Escuela de párvulos; experimento educacional; investigación pedagógica; docente de preescolar. (Palabras tomadas del Tesauro UNESCO).

\begin{abstract}
The research aimed to apply pedagogical strategies for boys and girls of initial education based on the Universal Design for Learning (UDL) in order to strengthen the intervention of the teacher in the teaching-learning processes of the C.E.I "Alonso Torres", through the planning of motivating activities. It was based from the qualitative approach with an ethnographic method in order to know and describe the events as they were generated in reality, according to the perspectives of the participants. The change that education requires is in the hands of teachers, because they must go hand in hand with new societies, it all depends on the attitude and desire to improve for the benefit of all children, through updates and training that allow the knowledge of new strategies such as how to apply them, improving educational quality.
\end{abstract}

Descriptors: Nursery schools; educational experiments; educational research; preschool teachers. (Palabras tomadas del Tesauro UNESCO).

\title{
INTRODUCCIÓN
}

El mundo actual avanza a pasos agigantados, el docente debe enfrentar el reto de comprender que sus estudiantes aprenden de distintas maneras; por tal motivo es necesario que los maestros avancen al ritmo de la sociedad actual, no solo con conocimientos de épocas pasadas, sino que debe ir actualizándose en nuevas metodologías y estrategias que cambien su manera tradicional de dar clases. Para ello, es necesario estar a la mira para verificar cuales son las necesidades de cada aprendiz del grado de Inicial para que cumplan sus actividades sin distracciones.

Para (Chiner-Sanz, 2011), en los países desarrollados, la inclusión y atención a la diversidad de estudiantes es de manera universal, proporcionando recursos y 
materiales a las Instituciones Educativas para que se cumpla el proceso de aprendizaje; sin embargo, este proceso varía entre países, unos están a un mejor nivel que otros en atención a las necesidades de sus estudiantes y por lo tanto tienen diversas maneras de velar por los estudiantes con algún tipo de discapacidad, pero de igual forma todos hacen lo posible para que se dé una verdadera inclusión en las aulas.

En América Latina, en la mayor parte de los países existe desigualdad en los aprendizajes de los estudiantes, ello ocasiona que no se llegan los objetivos propuestos, lo anteriormente expresado se ha visto evidenciado en los resultados que no han sido satisfactorios; la atención a la diversidad ha afectado principalmente a los niños de pueblos indígenas, mujeres, niños de bajos recursos económicos, afrodescendientes, personas que vienen de otros países entre otros, quienes han sido excluidos del logro igualitario en sus aprendizajes. Peor aún en estudiantes que tienen discapacidades en donde es más confuso el problema (Oficina Regional de Educación para América Latina y el Caribe (OREALC/UNESCO Santiago, 2013).

Los docentes piensan que atienden a la diversidad de estudiantes mediante el trato igualitario que les brindan en el aula de clases, pero al momento de planificar, al realizar las actividades con los niños, les dejan de lado haciendo otras actividades para ellos, excluyéndoles del trabajo que realizan el resto. En el aula de clases existe diversidad de aprendizajes, unos aprenden más rápido y otros más lento, por la diversa estructura del cerebro que todas las personas poseen y que difieren en la cantidad de espacio que ocupan, donde se hacen conexiones cuando aprendemos; por esta razón los estudiantes acceden de diferentes modalidades al aprendizaje y se expresan de diferentes formas (Pastor, Sánchez Serrano \& Zubillaga-del-Río, 2014).

Las escuelas y centros de educación deben atender a todo el contexto diverso de estudiantes e incluirlos al aprendizaje, favoreciendo al conocimiento, sin dejar de lado a educandos que tengan algún tipo de dificultad y que les impida de ser partícipes de estos procesos, creando de esta manera aprendizajes verdaderos (Sandoval-Mena, Simón- Rueda \& Echeita-Sarrionandia, 2012).

El estado ecuatoriano instaura a que todos los establecimientos de educación deben 
dar acceso a personas con discapacidad convirtiéndose en centros e instituciones de inclusión, donde los docentes traten por igual a los niños y niñas, eliminando barreras que impiden su aprendizaje y participación (Constitución de la República del Ecuador, 2008). La inclusión de estudiantes no solo debe ser el de insertarle al aula, donde la mayor parte de tiempo se utilizan textos o laminas que muchas veces son inaccesibles para niños que presentan problemas físicos, visuales y cognitivos (Rose, Meyer \& Hitchcock, 2005).

Es importante brindar a los estudiantes una educación inclusiva que ayude a la participación en los aprendizajes dejando atrás el hacerle a un lado; se debe volver a armar la cultura, políticas educativas, y las prácticas en los centros de aprendizaje en donde participen, para su progreso tanto en escuelas como la comunidad educativa para mejorar la calidad de la educación. Mediante la inclusión se puede minimizar barreras que impiden el aprendizaje, mediante la selección de recursos atendiendo a cada necesidad del estudiante (Booth \& Ainscow, 2002).

Los niños necesitan calidad de experiencias no solo a nivel familiar sino dentro del marco educativo donde los docentes deben brindarles eficacia en sus cuidados y adquisición de conocimientos, buscando alternativas y estrategias para mejor estos procesos; cuidar a los niños es una dura tarea, pero a la vez gratificante y agradable puesto que al estar en contacto con ellos hacen que la vida diaria sea más divertida.

Por otro lado, el (Ministerio de Educación del Ecuador, 2014) define que la Educación Inicial está conformada por niños de 3 a 5 años y está dividido en subniveles; el nivel 1 se encuentran los niños de 3 años y el nivel 2 conformada por niños de 4 años. (Peralta \& Fujimoto-Gómez, 1998), refieren que al hablar de niños de educación inicial, debe ser de acuerdo a sus necesidades e intereses para que se desarrollen de forma continua e integral, mediante labores que realice el docente de forma coordinada en diferentes experiencias para un aprendizaje significativo.

Por tal motivo es necesario establecer alternativas pedagógicas de acuerdo a diferentes factores sean estos culturales, socioeconómicas, y contextuales de los estudiantes; incorporando herramientas de trabajo que sean didácticas, metodológicas e 
innovadoras de inclusión, que permita considerar ritmos y necesidades de aprendizaje (Álvarez-Oquendo \& Chamorro-Benavides, 2018). Brindar una educación inclusiva que revolucione los sistemas y que aseguren la equidad entre todos los miembros con los mismos derechos y oportunidades (Sandoval-Mena, Simón-Rueda \& EcheitaSarrionandia, 2012).

Para atender a la diversidad de estudiantes y mejorar su participación en los procesos educativos, se ha implementado un enfoque junto a varios organismos y el Center for Applied Special Technology (CAST), los cuales han desarrollado un modelo llamado Diseño universal para el aprendizaje (DUA) que sirve como medio para eliminar barreras de aprendizaje de los estudiantes; mediante el acceso al currículo cambian los recursos, metodologías, adaptando a una evaluación flexible en base a principios que pretenden mejorar diferentes procesos en el aula de clase (Rose, Meyer \& Hitchcock, 2005).

Tener claro que son las estrategias de enseñanza es sumamente importante para poder emplearla dentro de la práctica docente, para (Díaz-Barriga \& Hernández-Rojas, 2002), son recursos que emplea el docente para cautivar la atención de sus estudiantes siendo incluidas antes, durante y después de la clase; lo primero que se debe de tomar en cuenta al momento de enseñar es relacionar la información nueva que la maestra presenta con lo que ellos conocían del tema; luego se debe presentar la información mediante ilustraciones visuales que tengan relación con el contenido para motivar al niño y por último es necesario conocer lo que aprendieron por medio de preguntas o un resumen.

Así mismo, (Gargallo- López, 2000; González, 2001; Beltrán-Llera, 2003), refieren que las estrategias de aprendizaje son un acumulado de recursos para que sean utilizados por los estudiantes para organizar los conocimientos de mejor manera y solucionar diversos problemas, además de poder dirigir sus propios aprendizajes. En este sentido, la presente investigación tuvo como objetivo aplicar estrategias pedagógicas para niños y niñas de educación inicial basadas en el DUA con la finalidad de fortalecer la intervención del docente en los procesos de enseñanza-aprendizaje del C.E.I "Alonso 
Torres", mediante la planificación de actividades motivadoras.

\section{Referencial teórico}

\section{Aplicaciones del DUA en el contexto internacional y nacional}

En este sentido, (Rose, Harbour, Daley, Johnston \& Abarbanell, 2006), así como un grupo de docentes utilizaron tres principios del Diseño Universal para el Aprendizaje en un curso impartido en la Universidad de Harvard, donde se desarrolló sitios web o Blogs creados para impartir textos, videos y grabaciones para acceder en cualquier momento; los estudiantes podían compartir sus aprendizajes e interactuar con su grupo en línea, publicar trabajos hechos con la creatividad de cada uno de ellos, por esta razón los autores valoran positivamente la implementación del DUA en el aula de clases.

En España, (Díez-Villoria \& Sánchez-Fuentes, 2015), refieren que el DUA podría disminuir las necesidades de adaptación de estudiantes especiales o con déficit de atención, por lo que es necesario la formación del profesorado en metodologías que atiendan a la diversidad. Por esta razón el autor sugiere a las universidades nuevas estrategias para afrontar esta problemática diseñando currículos inclusivos.

En Colombia (Moreno-Angarita et ál., 2016), adaptaron la visión del Diseño Universal para el Aprendizaje de grupo de Fonoaudiólogos que diseñaron e implementaron estrategias de acuerdo a las necesidades de todos los niveles del sistema educativo desde el Prescolar hasta la Universidad; los resultados favorecieron al acceso de información eliminando barreras mediante la aplicación de los tres principios del DUA a través de las siguientes fases: observación y caracterización, diseño y elaboración de estrategias, y recolección y análisis de datos.

Por otro lado, (Acosta-Sánchez, Bustamante-Tello \& Yuraszeck-Ardiles, 2016), analizaron estrategias motivacionales utilizadas por estudiantes de la carrera de parvulario de Chile que se autoevaluaron al momento de dar clases, según el análisis se entregó herramientas a los estudiantes para que puedan diseñar diversas estrategias motivacionales desde la perspectiva del Diseño Universal de Aprendizaje, sustentado en base de la neurociencia que permite cambios curriculares y flexibles para 
el trabajo en aula, mediante el factor motivacional que permite atender a la diversidad concluyendo que es posible cambiar los procesos educativos mediante interacciones positivas.

En prosecución a lo planteado, (Álvarez-Oquendo \& Chamorro-Benavides, 2017) en su proyecto sobre estrategias didácticas para la incorporación del DUA, en la escuela rural El Chiquí Colombia, describen que obtuvieron resultados de desmotivación de los estudiantes frente a los contenidos y metodologías empleadas por maestros de Inglés los cuales no utilizaban recursos tecnológicos y didácticos; luego de ello se reunieron el personal docente para proponer diferentes estrategias que ofrezcan diversas actividades, materiales y formas de evaluación.

Por su parte (Giraldo-Cardozo, Baldiris-Navarro \& Salas-Álvarez, 2017), en Colombia realizaron un curso de capacitación impartido a 44 egresados de la Universidad de Córdova para ser formadores y poder capacitar a 2672 docentes que no pertenecían al área de informática y que por lo que no contaban con bases suficientes para crear sus propios recursos digitales, el proyecto tuvo como finalidad el uso de las Tecnologías de Información y Comunicación (TIC) para mejorar estrategias de educación; se realizó desde el aprendizaje basado en problemas, donde incorporó al DUA para fortalecer las clases, los docentes a más de obtener conocimientos en las TIC podían crear ambientes inclusivos con buenas bases, estrategias y metodologías.

En el contexto ecuatoriano, (Otero, 2018) en su investigación en la Unidad Educativa Darío C. Guevara en Babahoyo, utilizó el DUA como estrategia para la implementación de herramientas tecnológicas en la asignatura de lengua y literatura, mediante el uso de medios audiovisuales para la comprensión lectora que permitieron encontrar ideas principales e interpretarlas. El autor recomienda que se aplique esta metodología de manera continua para mayor éxito en el aprendizaje.

De los estudios expuestos tanto nacionales como internacionales se han utilizado los principios del DUA en diferentes niveles educativos, manifestando estar de acuerdo con la propuesta, pues mejoraría procesos educativos atendiendo particularidades y diferenciando la diversidad, mediante la selección de recursos que llamen la atención y 
con la utilización de las TIC para su construcción. Es necesario transformar la educación mediante prácticas y estrategias inclusivas en todas las escuelas, aunque a veces resulte difícil y este lleno de retos, debido a las diversas realidades e individualidades de cada estudiante.

Se necesita que los docentes estén predispuestos al cambio para que la educación de un país cambie, porque de lo contrario se seguirá con las mismas metodologías de siempre que resulta para el estudiante monótono y con información que no asimilará. Para (Bodero-Arízaga, 2018) y (Silvia-Monturiol, 2019), es necesario trabajar con todos por igual y no dejar ningún estudiante excluido por tener alguna discapacidad, realizando todos las mismas actividades de tal manera que sean ricas en conocimientos.

\section{Influencia del DUA en el que hacer educativo}

En los años 90 los profesores de CAST sentían que el material que empleaban las escuelas como los libros eran similares a una escalera, es decir solo servían para unas personas, en la educación usar este tipo de materiales no brinda oportunidades de aprendizaje a estudiantes con discapacidad pues generan barreras en este proceso; de esta forma CAST desarrollo libros tecnológicos con diferentes opciones para que sean utilizados por estudiantes con discapacidad física o auditiva controlados por el habla. Después de varios trabajos CAST se reunió con un arquitecto llamado Ron Mace donde entablaron una conversación de como él construía utilizando el Diseño Universal que tenía varios beneficios para el acceso de personas, llegando a aplicarlo en Educación y creando el Diseño Universal para el Aprendizaje (DUA) (Rose, 1999).

Quién impulsó esta propuesta fue el Dr. David Rose de la Universidad de Harvard, describiendo que el DUA está dirigido a la diversidad de estudiantes, buscando eliminar las barreras que impiden el aprendizaje. Al principio se centró solo en estudiantes con discapacidad, pero luego fue dirigida a todos los que conforman el aula de clases (Moreno-Angarita, et ál., 2016). El DUA no solo se centra en que a los estudiantes se les incluya en el aula, sino que tengan las mismas oportunidades al momento de 
aprender, este modelo ayuda a que los docentes construyan currículos flexibles tanto en los objetivos de la clase como en los materiales, recursos y la forma de evaluación atendiendo a sus necesidades (Pastor, Zubillaga-del-Río \& Sánchez Serrano, 2015). En consideración, (Acosta-Sánchez, Bustamante-Tello \& Yuraszeck-Ardiles, 2016), destacan que el DUA se basa en aportes de las neurociencias para verificar como aprenden los estudiantes y la contribución de nuevas tecnologías como recursos para emplear en clases, además el constructivismo y el concepto de andamiaje de Vygotsky; la teoría de las inteligencias múltiples de (Gardner, 2015) que describe que hay tipos de inteligencia relacionándose mutuamente, predominan unas cuando el caso lo amerite, siempre estarán en constante cambio mientras las personas sigan adquiriendo información.

EI DUA describe que existen estudiantes que no alcanzan los aprendizajes planificados por sus maestros debido a que ellos aprenden de acuerdo a sus propios ritmos, posibilidades y limitaciones, en donde es necesario crear espacios que promuevan motivación, con recursos innovadores mediante la utilización de TIC donde el estudiante escoja el recurso que más le gusta de acuerdo a sus capacidades. Muchos docentes creen que los estudiantes aprenden con las mismas metodologías y no se dan en cuenta de que cada uno de ellos tienen diferentes estilos de recibir una información, pues unos aprenden viendo vídeos, escuchando canciones, realizando juegos y actividades manuales.

El (Ministerio de Educación del Ecuador, 2014), en su currículo de educación inicial reconoce que no todos los niños aprenden de la misma manera, ni al mismo ritmo, por eso el docente debe prestar interés a las necesidades de cada niño de su grupo mediante experiencias de aprendizaje que les cause motivación. Por consiguiente, (Pastor, Sánchez Serrano \& Zubillaga-del-Río, 2014), existen tres principios en el que se basa el DUA y son las siguientes:

- Red de reconocimiento, proporcionar múltiples formas de representación. Es el qué del aprendizaje, la manera de como los estudiantes perciben la información nueva, no se puede presentar una sola clase de contenidos sino 
variados con opciones para que los niños puedan ver, oír o utilizar el tacto.

- Red estratégica, proporcionar múltiples formas de acción y expresión. Es el cómo, los estudiantes demostrarán que han adquirido conocimientos mediante diferentes tareas en donde puedan expresar lo que ellos saben.

- Red afectiva, proporcionar múltiples formas de implicación. Es el porqué, todo el aspecto emocional influye en su aprendizaje y esto requiere que tengan una buena motivación, buscando diferentes maneras para que cambien su disposición al recibir nueva información.

El DUA tiene en cuenta los siguientes componentes para ser elaborados desde el currículo, las cuales son establecer las metas de hacia dónde quiero llegar con los estudiantes, que competencias se van a desarrollar, los métodos que voy a utilizar, las estrategias didácticas y pedagógicas; los materiales a utilizar, que son los recursos para dar clases; por último, la evaluación, donde se selecciona los medios a través de los cuales voy a evaluar (Movilla-Gastelbondo \& Suárez, 2019).

\section{El rol de las TIC en el DUA y en educación}

Desde que se empezó con el modelo del DUA, las tecnologías han tenido mucho que ver en su desarrollo, por el material que se podía conseguir para diferentes estudiantes que tenían necesidades que no eran iguales a las de sus compañeros, obteniendo resultados positivos gracias al uso de la tecnología, reemplazando a recursos de épocas pasadas que en su mayor cantidad eran impresos siendo muchos de estos materiales rígidos, sin flexibilidad por lo que no eran captados por todo el alumnado (Pastor, Zubillaga-del-Río \& Sánchez Serrano, 2015). Para (Rose, 1999) existen ventajas para la utilización de la tecnología digital gracias a las siguientes características:

- Versatilidad. La computadora mediante un software puede adquirir la función de un libro, reproducir videos, audios, hojas de cálculos, jugar videojuegos, etc. permitiendo combinar diferentes herramientas para las clases.

- Flexibilidad. En los medios digitales, gracias a que son flexibles se puede 
desarrollar softwares diferentes de acuerdo a las necesidades de los estudiantes.

- Capacidad para marcarlos. Permite transformar los contenidos e informaciones de acuerdo a lo que se requiera utilizando de diversas maneras.

- Capacidad para ponerlos en red. La información se puede relacionar con otras informaciones e ir actualizando con mayor rapidez.

Cabe recalcar que en los últimos años se ha visto que las tecnologías han decaído en su implementación dentro de las metodologías utilizadas, por la razón de que algunos no pueden manipularlas generando resistencia (Lombillo, et al., 2018). Mediante el DUA los estudiantes pueden a llegar a ser aprendices expertos que utilizan los conocimientos que adquirieron relacionándolos con nuevas informaciones, a fin de reestructurar en algo nuevo, teniendo en claro cuáles son los objetivos a alcanzar, reconociendo sus errores y sus fortalezas; para ellos deben ser alumnos decididos que no le teman al fracaso y que busquen aprender cosas nuevas para cumplir lo que se proponen (Pastor, et al., 2013).

Este modelo propone un cambio en la educación del estudiante para procurar aprendizajes significativos que duren desde los primeros años y durante toda su vida, invitando a los docentes a innovar, actualizarse y ver la mejor estrategia que beneficie a todos. El uso de estrategias basadas en el Diseño Universal para el Aprendizaje (DUA) va a permitir la participación en los procesos de enseñanza aprendizaje de todos los estudiantes permitiendo respetar su individualidad y su manera de aprender.

\section{MÉTODO}

El presente estudio utilizó un diseño no experimental con enfoque cualitativo y método etnográfico a partir de la convivencia de los investigadores en el ambiente natural donde se desarrolló la investigación (Aldana-Zavala, 2019), procesándose información a partir de entrevista grupal (Focus Group) a 11 docentes del CEl "Alonso Torres" y efectuada mediante una videoconferencia. Para posterior realizar una revisión bibliográfica en bases de datos especializadas encaminada a buscar estrategias pedagógicas para niños de Educación Inicial basados en el DUA, mismas que 
fortalezcan los procesos de enseñanza y aprendizaje, para posteriormente plantear su forma de aplicación en el aula de clase anclada a una planificación. (Rojas-Valdivieso, Erazo-Álvarez, Pozo-Cabrera, \& Narváez-Zurita, 2020).

\section{RESULTADOS Y APORTES GENERADOS}

El desarrollo de la entrevista se encaminó a conocer el cómo trabajan los docentes con niños, atendiendo a la diversidad en el aula de clases, se realizó una entrevista a la totalidad de maestros mediante el Focus Group realizado en línea, en la cual se obtuvieron los siguientes resultados:

Se consultó sobre ¿Cómo atiende a la diversidad de estudiantes en su aula? Algunos docentes respondieron que ellos brindan un trato igualitario sin discriminación alguna, otros mediante planificaciones aptas para todos y por último el resto de los docentes afirman que no es importante los conocimientos, sino más bien el brindar cariño y paciencia a todos los niños.

Se consultó también si ¿Ha trabajado alguna vez con estudiantes con discapacidad? Varios docentes afirman no haber trabajado con estudiantes con discapacidad, mientras que otros manifiestan que sí han trabajado con niños que presentan diversas discapacidades como autismo, parálisis cerebral, trastornos de lenguaje.

Otra consulta realizada fue ¿Cuáles son las dificultades de aprendizaje más comunes en su aula? La respuesta fue que todos están de acuerdo en que las mayores dificultades de los niños son los problemas en el lenguaje y falta de atención en clase, debido a que algunos niños se desconcentran con facilidad.

Con respecto a la pregunta ¿Qué actividades desarrollan en su planificación para que los niños con discapacidad sean incluidos en los aprendizajes? Los docentes argumentaron que hacen diferentes planificaciones para los niños con discapacidad con menor grado de complejidad haciendo que trabajen por separado, mientras el otro grupo trabaja con actividades normales que han planificado, es decir hacen adaptaciones curriculares.

Se consultó también si ¿Es difícil enseñar las mismas actividades a estudiantes con 
discapacidad? La totalidad de docentes afirma que es difícil trabajar con niños con discapacidad por lo que se hacen otras planificaciones.

Analizando las respuestas de los docentes algunas se contraponen, pues afirman dar un trato igualitario a todos los niños sin discriminación, sin embargo, realizan planificaciones separadas para su aula; no trabajan con las mismas actividades con los niños con discapacidad sino que realizan otras actividades con menor dificultad para ellos, excluyéndoles de la planificación, esto no significa que lo hacen intencionalmente, pero al no tener conocimientos sobre estrategias para aplicar a todos los niños, se les hace difícil encontrar un adecuado currículo.

Revisando las respuestas de la entrevista se ha visto la necesidad de aplicar los principios del Diseño Universal para el Aprendizaje y plantear estrategias que ayuden a incluir a los niños a las actividades regulares, principios que se detallan a continuación:

Principio I. Múltiples formas de representación, refiere a que la información debe ser perceptible para todos, proporcionado a través de los diferentes sentidos (Vista, Oído, tacto). En el proceso de enseñanza-aprendizaje se puede utilizar programas informáticos interactivos que sirven de apoyo en este proceso a través de diferentes recursos multimedia como videos, imágenes, audios y juegos que permiten que participe el niño despertando su interés gracias a la motivación que genera.

Se puede crear diversos recursos con tan solo utilizar programas como por ejemplo Educaplay, en donde se puede diseñar el material para trabajar en clases, mediante la plataforma YouTube se puede descargar diferentes videos, música, cuentos, etc. haciendo que las clases sean más motivadoras y que les cautiven a los niños logrando que estén predispuestos siempre a seguir aprendiendo. Los maestros debemos estar conscientes de que los niños están acostumbrados desde pequeños a convivir con el mundo digital en donde los gráficos, y contenidos multimedia hacen que aprendan y se sientan atraídos hacia ellos.

Los estudiantes deben ser atendidos y respetados individualmente porque todos son diferentes ya sea de acuerdo sus intereses, capacidades de memoria como la manera 
de trabajar en clase y de aprender; es ahí donde los centros educativos deben brindarles la misma oportunidad a todos de desarrollarse a su propio ritmo en un ambiente de colaboración mutua.

Las maestras deben caracterizar a su grupo de forma general y luego de forma individual, conociendo sus fortalezas y debilidades para de esa manera buscar y crear los recursos necesarios para el desarrollo del aprendizaje de su grupo. Es importante diseñar situaciones de aprendizaje de acuerdo a las necesidades de los estudiantes, partiendo de los conocimientos previos que ellos tienen para generar conocimientos y que puedan resolver sus problemas.

Los niños absorben todo lo que observan de su medio, por eso la necesidad de brindarles experiencias y materiales novedosos que le llamen la atención. No solo las personas aprendemos observando, otros aprenderán escuchando, sintiendo, tocando, manipulando por lo que propiciarles recursos y materiales adecuados para su grupo de estudiantes es muy importante.

Es necesario que toda la comunidad educativa esté presente en estos procesos en especial los padres de familia que también pueden ser partícipes de los aprendizajes de sus niños colaborando con las maestras de forma activa, generando bienestar emocional.

Principio II. Proporcionar múltiples formas de acción y expresión, mediante este principio los niños podrán expresar sus conocimientos de diferentes maneras ya sea de forma verbal o plasmando mediante su cuerpo de forma escrita o gráfica, por esta razón los maestros deben dar diversos materiales para que los estudiantes expresen lo que sienten y lo que saben por el medio que les resulte más fácil.

Para los niños una manera de pronunciarse es mediante el juego para establecer relaciones con otros niños y resolver los problemas que se le presentan. El juego debe ser libre, ordenado con intención a conseguir algo propuesto; los trabajos que diseñados por el maestro pueden ser sensoriales, motores o intelectuales. Todas estas actividades que el maestro realice debe ser para que los niños se impliquen en la 
construcción de sus aprendizajes y puedan expresarse o comunicarse de diversas maneras desenvolviéndose en su medio con seguridad y confianza.

El docente debe ser mediador en el aprendizaje de los niños y puede de esta forma trabajar diversas actividades mediante juegos de forma colaborativa, para que sientan motivación por seguir realizando las actividades; pueden descubrir para aprender experimentando y jugando para luego llevar a la práctica los conocimientos que aprendieron en la clase. Es importante que los niños reciban aprendizajes significativos, así podrán relacionar la información que ya existe en el pensamiento con nuevos conocimientos mediante recursos y materiales significativos.

Una de las mejores estrategias son las artes plásticas y artísticas que sirven para que los estudiantes puedan expresar sus sentimientos, pensamientos mediante la creatividad, la exploración y experimentación. Todo lo que aprenden los niños lo plasman en algo ya sea de forma verbal, musical o mediante actividades plásticas en donde utilizan todos los recursos a su alcance y construyen lo que saben porque son creativos. Otra de las formas para que los niños se expresen puede ser mediante el juego de roles, que son actividades lúdicas donde los niños participan de forma espontánea, pueden expresarse sin restricciones dando vuelo a su imaginación siendo muy beneficioso pues amplían su vocabulario al interactuar con sus compañeros.

Es muy importante brindar a los niños diferentes formas por donde puedan expresar sus conocimientos adquiridos, darles alternativas para que expresen lo que saben, en especial con los niños pequeños porque algunos pueden tener dificultades en su lenguaje, otros son más tímidos y no van a poder expresarse mediante la comunicación verbal, con otras alternativas ellos son capaces de lograr todo lo que se proponen.

Principio III. Proporcionar múltiples formas de implicación, detalla que la motivación que realizamos los maestros para que los estudiantes se involucren en los aprendizajes es importante porque si no existe la presencia de esta, habrá pérdida de interés, no hay una sola manera de implicarles a los aprendizajes, pero si el brindarles formas de participación sean estas de forma individual o grupal. 
La motivación es la principal estrategia del maestro a la hora de dar clases pues esta ayuda despertar el interés de estudiante en todo el momento del proceso de enseñanza-aprendizaje. En la motivación intervienen todos los elementos que son partes de la vida del estudiante, sus necesidades, intereses teniendo que ver en su desarrollo integral mediante las experiencias que reciben.

La motivación impulsa al estudiante a pensar y actuar positivamente, ya que genera emociones y sentimientos hacia algo determinado. Una buena persona que motiva es aquel que se puede comunicar, guiar, sugerir y ofrecer diversas alternativas a su grupo. Se debe motivar en todo el proceso de enseñanza aprendizaje y no solo al comienzo de la actividad, despertar el interés del estudiante por aprender por seguir queriendo más actividades que le enriquecerán el conocimiento y además generará sentimientos y emociones que le subirán el ánimo (Suaza-Atencio, 2020).

Si los niños se sientan bien consigo mismos y con su capacidad de aprendizaje lo reflejan en el momento de clases pues están predispuestos a participar en las diferentes estrategias que su maestra preparó. Los estudiantes que tienen dificultades de aprendizaje, muchas veces, la causa de sus problemas es la falta de motivación por lo que no pueden utilizar estrategias para su aprendizaje.

Para las actividades se pueden emplear grupos que sirvan para ayuda mutua o de forma individual, como el niño se sienta más cómodo. Trabajar grupalmente puede ayudar a que los niños ayuden a sus compañeros a guiarles a indicarles lo que talvez algo no entiendan o no puedan, pero no hay que obligarles, siempre hay que ver que el niño se sienta cómodo y gustoso.

La educación debe proporcionar al niño alegría y placer con información que le llame la atención, desarrollando en ellos la curiosidad, la emoción, dando temas de su interés caso contrario no le va a interesar. Los seres humanos estamos aprendiendo desde bebés y se sabe que cuando algún objeto lo desafía, lo reta más le llama la atención e intenta explorar todo para conocer el mismo (Páez, 2018).

Generar o provocar curiosidad es algo importante porque el niño va a querer seguir descubriendo cada vez más sobre el objetos o actividades y no va a ser necesario que 
la maestra intervenga, sino por sí solo va ir realizando hasta completar o saber que ha terminado su trabajo para pasar a la siguiente actividad.

En la siguiente tabla se evidencian en resumen como influyen los principios basados en el DUA para el desarrollo de las actividades a realizar en el proceso de enseñanzaaprendizaje y cumplir con los diferentes objetivos planteados:

\section{Tabla 1}

Principios del DUA en las actividades de enseñanza-aprendizaje

\begin{tabular}{|c|c|c|c|}
\hline ¿Qué? & ¿Cómo? & $\begin{array}{l}\text { Actividades } \\
\text { Motivantes }\end{array}$ & Resultados a obtener \\
\hline \multirow{3}{*}{$\begin{array}{c}\text { Diseño } \\
\text { Universal } \\
\text { para el } \\
\text { Aprendizaje }\end{array}$} & $\begin{array}{c}\text { PRINCIPIO } \\
I\end{array}$ & $\begin{array}{l}\text { Búsqueda de materiales } \\
\text { visuales auditivos que sean } \\
\text { interactivos y sensoriales. }\end{array}$ & $\begin{array}{lr}\text { Cumplir con los diferentes } \\
\text { objetivos } & \text { planteados, } \\
\text { además generar } \\
\text { comprensión de las }\end{array}$ \\
\hline & $\underset{\|}{\text { PRINCIPIO }}$ & $\begin{array}{l}\text { Como van hacer las } \\
\text { actividades e interactuar con } \\
\text { ellas y poder expresarlas de } \\
\text { diversas maneras. }\end{array}$ & $\begin{array}{l}\text { experiencias, motivación } \\
\text { por aprender, desarrollo } \\
\text { de la creatividad e } \\
\text { imaginación. }\end{array}$ \\
\hline & $\begin{array}{l}\text { PRINCIPIO } \\
\text { III }\end{array}$ & $\begin{array}{l}\text { Búsqueda de motivaciones } \\
\text { para implicar en los } \\
\text { aprendizajes durante todo el } \\
\text { proceso de la clase mediante } \\
\text { dinámicas, juegos, canciones, } \\
\text { bailes. }\end{array}$ & $\begin{array}{l}\text { Pensamiento crítico, } \\
\text { desarrollo del lenguaje } \\
\text { confianza y seguridad, } \\
\text { toma de decisiones, } \\
\text { trabajo individual y en } \\
\text { equipo. }\end{array}$ \\
\hline
\end{tabular}

Fuente: Elaboración propia

Para cumplir con los tres principios del DUA se elaboró una planificación con diferentes estrategias en donde se integran los tres principios para poder mejorar los procesos de enseñanza aprendizaje. Por lo tanto, primero es necesario conocer como está compuesto los elementos en una planificación que se detallará a continuación:

En educación inicial específicamente en el Ecuador se trabaja mediante la metodología del juego-trabajo que consisten en diseñar diferentes ambientes de aprendizaje con materiales adecuados llamados rincones, donde constan diferentes materiales para el uso de los niños (Ministerio de Educación del Ecuador, 2014). Para (Nielsen- 
Rodríguez, Romance-García \& Chinchilla-Minguet, 2019), los ambientes de aprendizaje son lugares de movimientos, acción y exploración interactiva de los estudiantes para su desarrollo integral, estos son diseñados para un fin determinado, además son espacios ordenados, motivadores para los niños donde pueden experimentar mediante diversas actividades.

Todo esto se realiza mediante el juego porque a los niños les encanta jugar y descubrir diferentes cosas ampliando su conocimiento. Las actividades que se realizan en los ambientes de aprendizaje tienen diferentes fines, pero siempre se articulan al objetivo general de la experiencia.

Además, se trabaja mediante experiencias de aprendizaje que son actividades retadoras cuyas ideas surgen de los estudiantes y en donde pueden realizar diversas actividades como; experimentar, descubrir, desarrollar su pensamiento para que sea creativo y construya sus aprendizajes mediante la relación con la realidad en la que viven y conocen (Ministerio de Educación del Ecuador, 2014), presentando además, los momentos de la experiencia de aprendizaje:

Momento de inicio (anticipación): En donde los niños dialogan y planean con la maestra que van a desarrollar para cumplir con los objetivos de la planificación.

- Momento de desarrollo (construcción): Es cuando los niños juegan mediante las actividades propuestas por su maestra, exploran, crean, experimentan, interactúan con los materiales y el docente es el mediador de su aprendizaje ayudándoles y respondiendo a sus inquietudes.

- Momento de cierre (consolidación): Se realiza al final de la clase donde los niños expresan lo que aprendieron, conversan lo que les gustó y los que les pareció difícil, llevando a la maestra a una retroalimentación y poder exponer los trabajos frente a sus familias o amigos (Ministerio de Educación del Ecuador, 2014).

Otro aspecto a considerar en la planificación es la evaluación, que, en educación infantil, mediante la información obtenida y su respectiva apreciación de todos los sucesos del aula y en los procesos de aprendizaje se encuentren soluciones a 
problemas que se presenten. La evaluación debe realizarse continuamente y de forma flexible por lo que requiere técnicas que para poder evaluar, entre estas están: la entrevista, la observación y el diálogo; también se necesita de instrumentos para poder recoger la información: ficha de matrícula, de entrevista, anecdotario, lista de cotejo, portafolio, escala de estimación, autoevaluación, informe formal cualitativo, descriptivo (Ministerio de Educación del Ecuador, 2014).

Utilizando toda la información que se revisó anteriormente, se puede planificar atendiendo a las necesidades de los estudiantes de tal manera que se pueda brindarles aprendizajes significativos. Las actividades están realizadas utilizando diferentes estrategias para diferentes ambientes con un tiempo límite de 45 minutos.

\section{Tabla 2.}

Estrategias basadas en el DUA aplicadas a la planificación

Nombre de la experiencia: Me gustan los animales

Objetivo general de la experiencia: Desarrollar las actividades en base a los tres principios del DUA para identificar las características de los animales mediante juegos, videos, canciones y movimientos corporales para representarlos y comprender los cuidados y protección que necesitan, generando sentimientos de ternura, solidaridad, y autonomía.

Ámbito: Relaciones con el medio natural y cultural Rincón: Ciencias

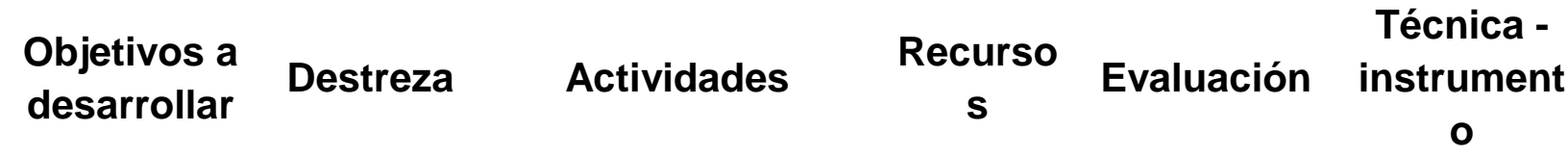

-Presentar

mediante

diversos

medios

información

sobre los

animales

salvajes

domésticos.
Anticipación.

Identificar -Observar el video de TV las animales domésticos DVD

caracterís y salvajes y describe $\mathrm{Cd}$ ticas de los animales que Parlante aparecieron en el USB cuento y que hicieron animales cada uno de ellos.

y doméstic os y -Conversar a cerca
Identifica las característica Técnica $s$ de los Observació animales $n$ domésticos y Instrument silvestres o: Lista de estableciend cotejo.

o la 


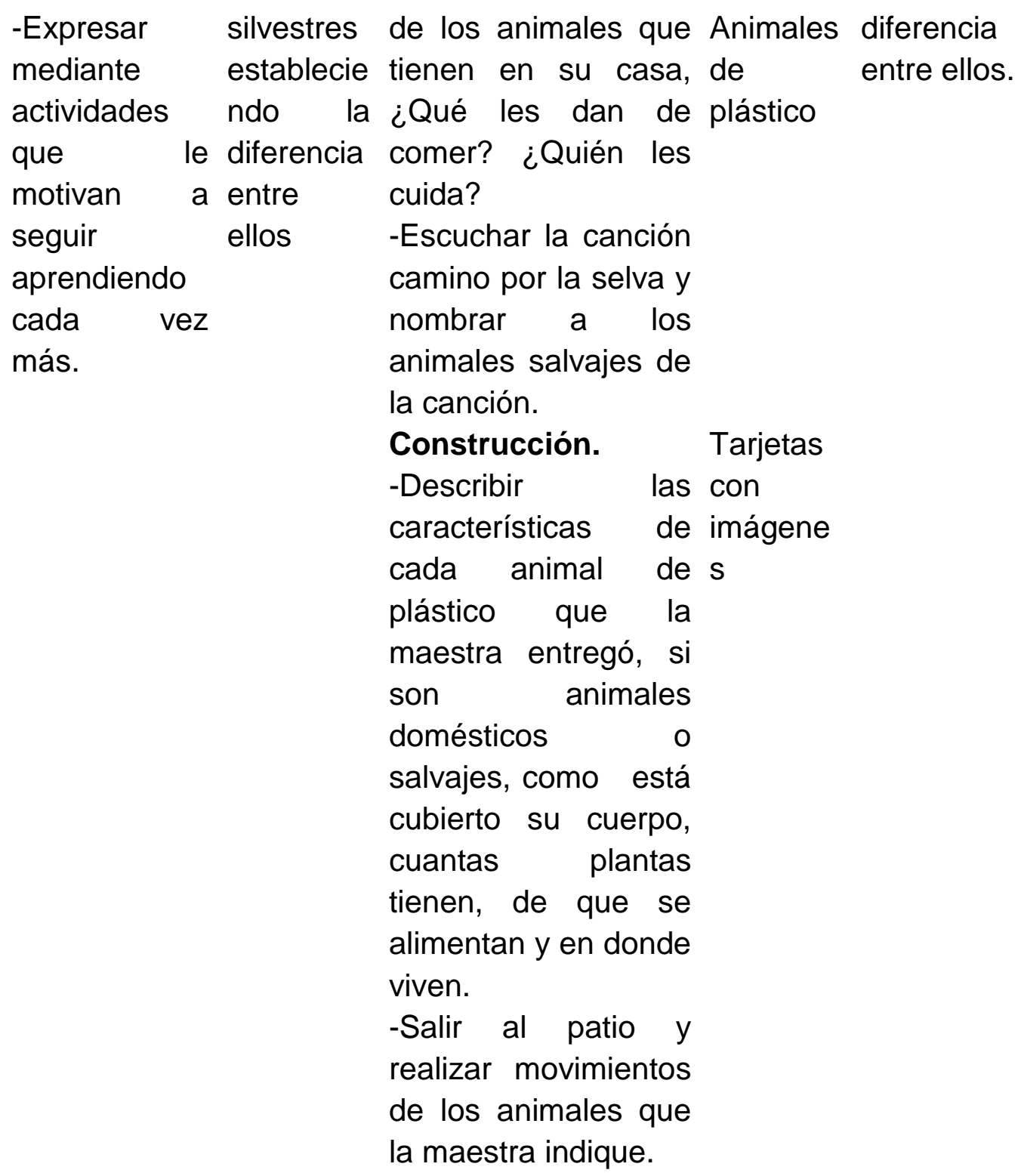

\section{Consolidación}

-Adivinar mediante

un juego de tarjetas

que animal es, dando

algunas de las

características.

-Jugar a descubrir a que animal 


$\begin{array}{ll}\text { corresponde } & \text { el } \\ \text { sonido que la } \\ \text { maestra emita. }\end{array}$

Ámbito: Lógico Matemáticas

-Presentar

variada

información

para que

aprendan a

contar los

números

relacionando

con

el Contar

nombre de la oralmente

experiencia de

en múltiples

formatos

15

1 al secuencia a

tanto para

ver, escuchar

$y$ tocar.

-Permitir que

elijan las

actividades

de evaluación

generando

autonomía,

seguridad $y$

confianza.

-Presentar

actividades

motivadoras

que le llamen

la atención al

niño para

involucrarse

\section{Anticipación}

-Observar mediante TV

un video "Números Dvd

para contar, Cd

animales del Zoo" Parlante Cuenta

para poder describir USB

que animales

aparecen y contar

cuantos hay.

-Escuchar la canción

"Cuenta y muévete" Animales

de los números del 1 de

al 15 y contar de plástico.

acuerdo al ritmo de Patio con

la canción.

dibujos

Construcción

-Observar los dibujos animales

de los animales que.

se encuentran en el

patio y saltar encima

de las imágenes

contando cuantos Caja de

hay.

madera.

-Formar grupos para Huevos

contar diferentes de

animales de plástico plástico.

que la maestra le Lamina.

proporciona

y Cinta

describe

animales tiene.

que doble

Consolidación.
Rincón: Construcción

Técnica:

Observació

$\mathrm{n}$.

Instrument oralmente cotejo.

del 1 al 15

con

secuencia

numérica. o: Lista de 
en las

actividades.
Ámbito: Expresión artística

\begin{tabular}{|c|c|c|c|c|c|}
\hline Ámbito: Expre & sión artístic & & Rin & cón: Artes & \\
\hline Presentar & Expresar & Anticipación & Tv & Expresa sus & Técnica: \\
\hline actividades & sus & -Observar & DVD & vivencias $y$ & Observació \\
\hline donde & vivencias & Escuchar la música" & $\mathrm{Cd}$ & experiencias & \\
\hline puedan & & El baile de los & Instrume & a través del & Instrument \\
\hline expresar sus & experienc & animales" y con sus & ntos & dibujo libre. & o: Lista de \\
\hline conocimiento & ias a & amigos canta y baila & musicale & & cotejo. \\
\hline s mediante & través del & la canción. & $\mathrm{S}$ & & \\
\hline diversas & dibujo & -Formar & Maracas & & \\
\hline $\begin{array}{l}\text { actividades. } \\
\text {-Permitir que }\end{array}$ & libre. & $\begin{array}{l}\text { orquesta con los } \\
\text { niños donde tienen }\end{array}$ & $\begin{array}{l}\text { Tambore } \\
\text { s }\end{array}$ & & \\
\hline se expresen & & que entonar la & Campan & & \\
\hline mediante las & & canción de "La & as & & \\
\hline artes & & orquesta de los & Flautas & & \\
\hline plásticas & & animales" utilizando & Lamina & & \\
\hline musicales & & instrumentos & A3 & & \\
\hline generando & & musicales. & Hojas & & \\
\hline creatividad e & & Construcción & secas & & \\
\hline imaginación. & & -Organizar grupos de & Ramas & & \\
\hline -Presentar & & trabajo y entregar & Hierva & & \\
\hline materiales & & láminas $\mathrm{A} 3$ a cada & Crayone & & \\
\hline para motivar & & niño para que & $S$ & & \\
\hline a usar su & & dibujen un animal de & Tempera & & \\
\hline imaginación y & & su preferencia como & s & & \\
\hline creatividad. & & $\begin{array}{l}\text { ellos lo imaginan. } \\
\text {-Decorar a su animal }\end{array}$ & $\begin{array}{l}\text { Papel de } \\
\text { seda }\end{array}$ & & \\
\hline
\end{tabular}

Elige cuál de las dos recortad actividades quiere os.

realizar:

-Contar cuantos

huevos hay en los

nidos de gallina

hechas de tabla.

- Pegar en la lámina

A3 animales de la

granja y contar

cuantas pegó. 


con material del Goma
medio como ramas y Papel de
hojas secas, hierba o brillo
con recursos del aula Caritas
como pinturas, felices
crayones, temperas,
bolitas de papel de
seda, trozos de
papel.

\section{Consolidación}

-Exhibir sus trabajos

$y$ describir que

animal dibujo $y$

decoró.

-Recibir caritas

felices por sus

trabajos.

Fuente: Elaboración propia

\section{Tabla 3}

Relación de los principios del DUA en las actividades planificadas

\section{Relación}

\section{Múltiples formas de presentación}

En el momento de anticipación está presente el primer principio:

- Se presentó materiales de auditivos y visuales e imágenes.

- Se dialogó con los niños acerca de la información presentada.

- Se realizaron preguntas acerca de lo que observaron.

\section{Múltiples formas de expresión}

En la planificación realizada en los momentos de construcción y en consolidación en la está presente este principio:

- Se ofrece la posibilidad de interactuar con los materiales presentados mediante la exploración sensorial.

- Los niños eligieron los materiales para trabajar fomentando la creatividad y participación.

- Ofrece la posibilidad de trabajo individual y grupal como el niño más se acomode. 


\section{Múltiples formas de implicación}

Esta actividad está presente en los tres momentos: Anticipación, Construcción, Consolidación.

- Se brinda materiales llamativos y diferentes que promuevan su atención.

- Las actividades eran desarrolladas pensando en todos los niños del aula porque a algunos les gusta aprender viendo videos, imágenes, a otros les gusta la música, y a otros les gusta realizar actividades manuales, motrices para el desarrollo de la experiencia.

\section{Fuente: Elaboración propia}

Estas estrategias elaboradas pretenden que todos los niños se involucren a las actividades, que anteriormente no lo podían hacer porque había pocas actividades, en algunos casos no se podía ver, escuchar y sentir mediante la percepción. Mediante estas actividades se pueden expresarse diversas formas, se realizaron con materiales novedosos, atractivos que le llamen la atención, que le provoquen curiosidad para que sigan descubriendo nuevas maneras y formas de aprender (Roque-Díaz, 2018).

Los aprendizajes que reciben los niños deben ser sostenibles duraderos en donde todos se sientan bien, confíen en sus conocimientos y puedan aplicar estos para ir construyendo durante toda su vida (Vallejo-Valdivieso, Zambrano-Pincay, VallejoPilligua \& Bravo-Cedeño, 2019), abordando las necesidades de todos los alumnos, viendo su individualidad para fortalecer su desarrollo integral mediante una enseñanza estructurada; es necesario que los docentes vayan al mismo ritmo es aspectos de tecnología para afrontar nuevos retos educativos.

En los primeros años de la infancia es fundamental permitir a los niños forjar las bases para su desarrollo futuro, es necesario brindarles una educación de calidad enmarcadas en las experiencias de aprendizaje, las cuales deben ser innovadoras que busquen el beneficio de todos los niños sin excluirles a otros, talvez por su discapacidad o por el hecho de su contexto diverso.

Mediante la fundamentación teórica se ha podido profundizar en los diferentes aportes del Diseño Universal para el Aprendizaje (DUA) y sus benéficos en el ámbito educativo permitiendo utilizar estos principios para atender a la diversidad de estudiantes de las 
aulas de clases y poder crear aprendizajes flexibles enmarcadas a la atención de las necesidades e intereses de los niños para que puedan recibir aprendizajes significativos.

Los niños perciben la información mediante su vista, oído, tacto y es ahí donde se deben presentar materiales novedosos que le llamen la atención (Reyes-Aular, 2017), pues esto les permitirá descubrir por si solos nuevos conocimientos de tal manera que se puedan expresar de diferentes maneras de forma libre, dejando que escoja el medio por el que más se sienta cómodo. Las actividades y estrategias que se utilicen siempre deben provocar en los niños curiosidad por aprender, mientras los niños más motivados se sientan con los materiales y estrategias utilizadas por los docentes, su actitud al aprendizaje podrá mejorar.

Es fundamental brindarles la oportunidad de interactuar con diferentes actividades pensando en todo el grupo de estudiantes siempre conscientes de que son diferentes no solo en su aspecto físico sino intelectual, que tienen fortalezas y debilidades que deben ser atendidas de forma prioritaria, por tal motivo es muy importante elaborar estrategias que atiendan a todos los estudiantes del aula generando en ellos diversión y alegría por aprender (Romero-Ramírez, 2020).

El cambio que requiere la educación está en las manos de los docentes, pues ellos deben ir a la par con las nuevas sociedades, todo depende de la actitud y el deseo de mejorar en beneficio de todos los niños y niñas, mediante actualizaciones $y$ capacitaciones que permitan el conocimiento de nuevas estrategias como la forma de aplicarlas mejorando la calidad educativa.

\section{FINANCIAMIENTO}

No monetario.

\section{AGRADECIMIENTO}

Al personal directivo y docente del CEI Alonso Torres por apoyaron la investigación. 


\section{REFERENCIAS CONSULTADAS}

Aldana-Zavala, J. J. (2019). La competencia epistemológica en el investigador social universitario venezolano. [Epistemological competence in the Venezuelan university social researcher]. Praxis, 15(1), 103-115. https://doi.org/10.21676/23897856.3091

Acosta-Sánchez, M., Bustamante-Tello, J., \& Yuraszeck-Ardiles, J. (2016). Estrategias motivacionales para ser utilizadas desde el enfoque del diseño universal de aprendizaje DUA, por estudiantes en práctica profesional de la carrera de educación parvularia del instituto profesional IPChile, en el desarrollo curricular de las experiencias de enseñanza - aprendizaje implementadas en el aula, con niños y niñas de nivel transición i y ii enjardines infantiles de la comuna de Puente Alto y San Miguel. [Motivational strategies to be used from the approach of the universal design of DUA learning, by students in professional practice of the kindergarten education career of the professional institute IPChile, in the curricular development of the teaching-learning experiences implemented in the classroom, with children and girls of transition level $\mathrm{i}$ and ii kindergartens of the commune of Puente Alto and San Miguel]. Recuperado de https://n9.cl/29pu

Álvarez-Oquendo, R., \& Chamorro-Benavides, D. (2018). Estrategias didácticas para la incorporación del diseño universal para el aprendizaje en la escuela rural. [Didactic strategies for incorporation of universal design for learning in rural school]. Revista $\quad$ Panorama, 11(21), 68-81. http://dx.doi.org/10.15765/pnrm.v11i21.1061

Beltrán-Llera, J. (2003). Estrategias de aprendizaje. [Learning strategies]. Revista de educación, 332, 55-73.

Bodero-Arízaga, L. (2018). Carencia de la Metodología Pedagógica en la Educación Inclusiva en Ecuador. [Lack of Pedagogical Methodology in Inclusive Education in Ecuador]. Espirales, 2(16), 112-135.

Booth, T, \& Ainscow, M. (2002). Guía para la evaluación y mejora de la educación inclusiva. [Guide for the evaluation and improvement of inclusive education]. Consorcio Universitario para la Educación Inclusiva. Recuperado de https://n9.cl/dn3li

Constitución de la República del Ecuador (2008). Título II. Derechos. Sección Quinta. Educación. [Title II. Rights. Fifth Section. Education]. Recuperado de https://n9.cl/sia 
Chiner-Sanz, E. (2011). Las percepciones y actitudes del profesorado hacia la inclusión del alumnado con necesidades educativas especiales como indicadores del uso de prácticas educativas inclusivas en el aula. [Teachers' perceptions and attitudes towards the inclusion of students with special educational needs as indicators of the use of inclusive educational practices in the classroom]. Universidad de Alicante. Recuperado de https://n9.cl/sosw

Díaz-Barriga, F., \& Hernández-Rojas, G. (2002). Estrategias docentes para un aprendizaje significativo. Una interpretación constructivista. [Teaching strategies for meaningful learning. A constructivist interpretation]. Mc Graw Hill Interamericana Editores, S.A. de C.V.

Díez-Villoria, E., \& Sánchez-Fuentes, S. (2015). Diseño universal para el aprendizaje como metodología docente para atender a la diversidad en la universidad. [Universal design for learning as a teaching method in order to meet the need for diversity in universities]. Aula abierta, 43(2), 87-93. https://doi.org/10.1016/j.aula.2014.12.002

Gardner, H. (2015). Inteligencias múltiples: La teoría en la práctica. [Multiple Intelligences: Theory in Practice]. PAIDÓS. Barcelona.

Gargallo- López, B. (2000). Procedimientos: Estrategias de aprendizaje: Su naturaleza, enseñanza y evaluación. [Procedures: Learning strategies: Its nature, teaching and evaluation]. Tirant lo Blanch.

Giraldo-Cardozo, J., Baldiris-Navarro, S., \& Salas-Álvarez, D. (2017). Diseño e implementación de recursos educativos digitales abiertos inclusivos. [Design and implementation of inclusive open digital educational resources]. Revista $Q, 9(18)$. $1-21$.

González, V. (2001). Estrategias de enseñanza y aprendizaje. [Teaching and learning strategies]. Editorial Paz. México: Librería Carlos Cesarman, S.A.

Lombillo-Rivero, I, Nambalo-Mulay-Dua, J, Torres-Alonso, A, \& Pérez-Hernández, B. (2018). La innovación educativa en el uso de los medios de enseñanza: una propuesta de solución que incluye las TIC. [Educational innovation in the use of teaching aids: a solution proposal that includes ICT]. Revista Cubana de Educación Superior, 37(3), e12. Recuperado de https://n9.cl/ed7u

Ministerio de Educacion del Ecuador (2014). Currículo de Educacion Inicial. [Currículo de Educacion Inicial]. Recuperado de https://n9.cl/47zr 
Ministerio de Educacion del Ecuador (2015). Reglamento General a la Ley Organica de Educación Intercultural. [General regulations to the Organic Law of Intercultural Education]. Recuperado de https://n9.cl/bca5

Movilla-Gastelbondo, I., \& Suárez, S. (2019). El diseño universal del aprendizaje (DUA): una estrategia pedagógica para la cualificación de la intervención docente en el marco de la escuela inclusiva. [The universal design of learning (DUA): a pedagogical strategy for the qualification of teaching intervention in the framework of the inclusive school]. Recuperado de https://n9.cl/aufv

Moreno-Angarita, M, Murillo-Avellaneda, A, Padilla-Quiroga, G, Albarracín-Garay, B, Pinzón-Fajardo, M, Bernal-Gómez, Y, Merchán-Ruiz, L, Puentes-Bernal, A, \& Riberos, L. (2016). Estrategias pedagogicas basadas en el diseño universal para el aprendizaje: un aproximación desde la comunicación educativa. [Pedagogical strategies based on universal design for learning: an approach from educational communication].Recuperado de https://n9.cl/wsz0

Nielsen-Rodríguez, A., Romance-García, Á., \& Chinchilla-Minguet, J. (2019). Los ambientes de aprendizaje como metodología activa promotora de la actividad física en Educación Infantil. Un estudio de caso. [Learning environments as an active methodology to promote physical activity in Early Childhood Education. A case study]. Retos, 37(37), 498-504. Recuperado de https://n9.cl/yisqn

Oficina Regional de Educación para América Latina y el Caribe (OREALC/UNESCO Santiago (2013). Situación Educativa de América Latina y el Caribe: Hacia la educación de calidad para todos al 2015. Recuperado de https://n9.cl/22t6

Otero, J. (2018). Planificación e implementación de una unidad didactica de lengua y literatura teniendo en cuenta el diseño universal del aprendizaje. [Planning and implementation of a didactic unit of language and literature taking into account the universal design of learning]. Recuperado de https://n9.cl//531

Páez, A. (2018). Docentes y padres en el proceso de aprendizaje de los estudiantes. [Teachers and parents in the student learning process]. EPISTEME KOINONIA, 1(2), 18-34. http://dx.doi.org/10.35381/e.k.v1i2.509

Pastor, C., Zubillaga-del-Río, A., \& Sánchez Serrano, J. (2015). Tecnologías y Diseño Universal para el Aprendizaje (DUA): experiencias en el contexto universitario e implicaciones en la formación del profesorado. [Technology and Universal Design for Learning (UDL): experiences in the university context]. RELATEC, 14(1), 89100. https://doi.org/10.17398/1695-288X.14.1.89 
Pastor, A., Sánchez-Hípola, P, Sánchez-Serrano, J. \& Zubillaga-del-Río, A. (2013). Pautas sobre el Diseño Universal para el Aprendizaje (DUA) Texto Completo (Versión 2.0). [Universal Design for Learning (DUA) Guidelines Full Text (Version 2.0)]. Recuperado de https://n9.cl/o8qk

Pastor, A., Sánchez Serrano, J. \& Zubillaga-del-Río, A. (2014). Diseño Universal para el Aprendizaje (DUA) Pautas para su introducción en el currículo. [Universal Design for Learning (DUA) Guidelines for its introduction in the curriculum]. Recuperado de https://n9.cl/jw0h

Peralta, V., \& Fujimoto-Gómez, G. (1998). La atención integral de la primera infancia en América Latina: ejes centrales y los desafíos para el siglo XXI. [Comprehensive early childhood care in Latin America: central axes and challenges for the $21 \mathrm{st}$ century]. Recuperado de https://n9.cl/wor9

Reyes-Aular, M. (2017). Formación Pedagógica dirigida a las madres, padres y representantes para el fortalecimiento del aprendizaje de los niños del PERE 191. [Pedagogical Training aimed at mothers, fathers and representatives for strengthening the learning of children of PERE 191]. Revista Arbitrada Interdisciplinaria Koinonía, 2(3), 109-130. Recuperado de https://fundacionkoinonia.com.ve/ojs/index.php/revistakoinonia/article/view/55

Romero-Ramírez, M. (2020). Vocación docente como respuesta esperanzadora en contextos de vínculos humanos frágiles. [Teaching vocation as a hopeful response in contexts of fragile human ties]. EPISTEME KOINONIA, 3(5), 27-50. http://dx.doi.org/10.35381/e.k.v3i5.527

Roque-Díaz, R. (2018). Desarrollo personal y manejo asertivo de emociones en estudiantes. [Personal development and assertive management of emotions in $\begin{array}{llll}\text { students]. } & \text { EPISTEME }\end{array}$ http://dx.doi.org/10.35381/e.k.v1i2.511

Rojas-Valdivieso, M., Erazo-Álvarez, J., Pozo-Cabrera, E., \& Narváez-Zurita, C. (2020). Prueba en Garantías Jurisdiccionales. Falta de regulación y afección al derecho a la defensa y libertad probatoria en el Ecuador. [Evidence on Jurisdictional Guarantees. Lack of regulation and infringement of the right to defense and evidentiary freedom]. IUSTITIA SOCIALIS, 5(8), 28-49. http://dx.doi.org/10.35381/racji.v5i8.560

Rose, D. (1999). Universal Design for Learning. [Diseño universal para el aprendizaje].Journal of Special Education Technology, 15(1), 67-70. https://doi.org/10.1177/016264340001500108 
Rose, D., Harbour, W., Daley, S., Johnston, C., \& Abarbanell, L. (2006). Harbour, Universal design for learning in postsecondary education: Reflections on principles and their application. [Diseño universal para el aprendizaje en la educación postsecundaria: reflexiones sobre los principios y su aplicación]. Journal of postsecondary education and disability, 19(2), 135-151.

Rose, D., Meyer, A., \& Hitchcock, C. (2005). The universal design classroom: accessible curriculum and digital technologies. [El aula de diseño universal: currículo accesible y tecnologías digitales]. Harvard Education Press.

Sandoval-Mena, M., Simón-Rueda, C., \& Echeita-Sarrionandia, G. (2012). Análisis y valoración crítica de las funciones del profesorado de apoyo desde la educación inclusiva. [Analysis and Critical Evaluation of Support Teachers' Functions from the Standpoint of Inclusive Education]. Revista de educación. Número extraordinario 2012, 117-137. doi:DOI: 10.4438/1988-592X-RE-2012-EXT-209

Silvia-Monturiol, F. (2019). Ponencia de Vásquez, Ana Patricia. Des-aprender para aprender. [Presentation by Vásquez, Ana Patricia. Unlearning to learn]. Recuperado de https://n9.cl/4rbk

Suaza-Atencio, E. (2020). Métodos de Aprendizaje para el Desarrollo de la Dimensión Estética en Estudiantes de Educación Inicial. [Learning Methods for the Development of the Aesthetic Dimension in Early Childhood Students].CIENCIAMATRIA, 6(1), 84-101. https://doi.org/10.35381/cm.v6i1.296

UNESCO (2016). XI y XII Jornadas de Cooperación Educativa con Iberoamérica sobre Educación Especial e Inclusión Educativa. [XI and XII Conference on Educational Cooperation with Ibero-America on Special Education and Educational Inclusion]. Recuperado de https://n9.cl/2ux8

Vallejo-Valdivieso, P., Zambrano-Pincay, G., Vallejo-Pilligua, P., \& Bravo-Cedeño, G. (2019). Estructuras mentales en la construcción de aprendizaje significativo. [Mental structures in the construction of meaningful learning]. CIENCIAMATRIA, 5(8), 228-241. https://doi.org/10.35381/cm.v5i8.257 